\title{
Young and Especially Senescent Endothelial Microvesicles Produce NADPH: The Fuel for Their Antioxidant Machinery
}

\author{
Guillermo Bodega $\mathbb{D}^{1}$, Matilde Alique, ${ }^{2}$ Lourdes Bohórquez, ${ }^{2}$ Miriam Morán, ${ }^{2}$ \\ Luis Magro, ${ }^{2}$ Lilian Puebla, ${ }^{2}$ Sergio Ciordia, ${ }^{3}$ María C. Mena, ${ }^{3}$ Elvira Arza, ${ }^{4}$ \\ and Manuel R. Ramírez ${ }^{2}$ \\ ${ }^{1}$ Departamento de Biomedicina y Biotecnología, Universidad de Alcalá, Alcalá de Henares, 28805 Madrid, Spain \\ ${ }^{2}$ Departamento de Biología de Sistemas, Universidad de Alcalá, Alcalá de Henares, 28805 Madrid, Spain \\ ${ }^{3}$ Proteomics Facility, Centro Nacional de Biotecnología, CSIC, Campus de Cantoblanco, 28049 Madrid, Spain \\ ${ }^{4}$ Unidad Técnica de Microscopía, CNIC, Cl Melchor Fernández Almagro 3, 28029 Madrid, Spain
}

Correspondence should be addressed to Guillermo Bodega; guillermo.bodega@uah.es

Received 13 December 2017; Revised 14 February 2018; Accepted 25 February 2018; Published 5 April 2018

Academic Editor: Claudio Cabello-Verrugio

Copyright (c) 2018 Guillermo Bodega et al. This is an open access article distributed under the Creative Commons Attribution License, which permits unrestricted use, distribution, and reproduction in any medium, provided the original work is properly cited.

\begin{abstract}
In a previous study, we demonstrated that endothelial microvesicles (eMVs) have a well-developed enzymatic team involved in reactive oxygen species detoxification. In the present paper, we demonstrate that eMVs can synthesize the reducing power $(\mathrm{NAD}(\mathrm{P}) \mathrm{H})$ that nourishes this enzymatic team, especially those eMVs derived from senescent human umbilical vein endothelial cells. Moreover, we have demonstrated that the molecules that nourish the enzymatic machinery involved in $\mathrm{NAD}(\mathrm{P}) \mathrm{H}$ synthesis are blood plasma metabolites: lactate, pyruvate, glucose, glycerol, and branched-chain amino acids. Drastic biochemical changes are observed in senescent eMVs to optimize the synthesis of reducing power. Mitochondrial activity is diminished and the glycolytic pathway is modified to increase the activity of the pentose phosphate pathway. Different dehydrogenases involved in NADPH synthesis are also increased. Functional experiments have demonstrated that eMVs can synthesize NADPH. In addition, the existence of NADPH in eMVs was confirmed by mass spectrometry. Multiphoton confocal microscopy images corroborate the synthesis of reducing power in eMVs. In conclusion, our present and previous results demonstrate that eMVs can act as autonomous reactive oxygen species scavengers: they use blood metabolites to synthesize the $\mathrm{NADPH}$ that fuels their antioxidant machinery. Moreover, senescent eMVs have a stronger reactive oxygen species scavenging capacity than young eMVs.
\end{abstract}

\section{Introduction}

Extracellular vesicles are subcellular structures produced by many different cells and located in the extracellular compartment. There are diverse types of extracellular vesicles depending on their biogenesis, content, function, and/or biophysical properties $[1,2]$. The most common classification includes three types: microvesicles (MVs) (also termed microparticles or ectosomes), exosomes, and apoptotic bodies [3]. MVs are released from the cell by the budding of the plasma membrane and represent not only a heterogeneous structural population, since their size usually ranges from 0.15 to $1 \mu \mathrm{m}$ or more, but also a heterogeneous functional group. They are present in all the body fluids: urine, saliva, bile, amniotic liquid, synovial, cerebrospinal, and seminal fluids as well as blood. Plasma MVs are of diverse cellular origin; they can arise from endothelial cells, erythrocytes, platelets, and leucocytes [4]. Blood MVs have been involved in the regular maintenance of endothelial cells [5] and other physiological functions such as coagulation, reticulocyte maturation, and angiogenesis [3] as well as mediators of endothelial dysfunction [6]. They have also been used as therapeutic tools in cardiovascular disease [7].

Reactive oxygen species (ROS) are produced as a consequence of cell metabolism, although the cells contain different mechanisms to eliminate them. In fact, the oxidative stress 
caused by an increase of ROS can be considered as an imbalance between ROS production and elimination. Although ROS have physiological roles in the regulation of vascular cell function [8], vascular formation, and development [9], this imbalance has been associated with aging and senescence [10-14] as well as other pathological conditions of the cardiovascular system [15-17], more specifically, with endothelial cell dysfunction [18-24].

$\mathrm{NADPH}$ is, obviously, closely related to NADH, another molecule with a reducing activity. Both may act as common mediators in different biological processes. Hence, it is common to find the term $\mathrm{NAD}(\mathrm{P}) \mathrm{H}$, indicating that both molecules may be involved. Nevertheless, $\mathrm{NAD}^{+}$is mainly involved in catabolic reactions and mitochondrial functions, whereas $\mathrm{NADP}^{+}$is involved in cellular antioxidant systems and anabolic reactions [25]. In fact, NADPH is considered the sole source of reducing power of antioxidant systems [26]. This is the reason why NADPH is considered the "fuel" of the antioxidant machinery. We have recently demonstrated that endothelium-derived MVs (eMVs) have a well-developed and functional enzymatic team to eliminate ROS which, moreover, increases in senescence [27]. Apparently, if eMVs can eliminate ROS, they should (a) contain large amounts of NADPH, (b) obtain it from the plasma, or (c) be able to synthesize it. Thus, the aim of this work was to elucidate these questions. In addition, given the differences observed in our previous study, where senescent eMVs showed a higher capacity of ROS elimination than young eMVs, we have also analyzed the capacity of eMVs obtained from both young and senescent endothelial cells to produce NADPH.

\section{Material and Methods}

2.1. HUVEC Culture. Cryopreserved human umbilical vein endothelial cells (HUVECs) (ATCC Cat number PCS-100010) were cultured in endothelial growth medium (Lonza) supplemented with $10 \%$ heat-inactivated foetal bovine serum (Sigma-Aldrich). Cultures were maintained at $37^{\circ} \mathrm{C}$ in a $5 \%$ $\mathrm{CO}_{2}$ atmosphere at $95 \%$ humidity. The HUVECs were serially passaged (the replicative senescence model). Cells passaged $<8$ times (population doubling $(\mathrm{PD})<20$; with $\mathrm{PD}$ calculated as $[\ln \{$ number of cells harvested $\}-\ln \{$ number of cells seeded $\} / \ln 2]$ ) were regarded as young endothelial cells, while those passaged 27-35 times (PD > 96) were regarded as senescent [28]. The proliferation rate of the latter cells is remarkably reduced, and more than $70 \%$ are positive for senescence-associated $\beta$-galactosidase. Prior to use, HUVEC extracts from cells passaged 4-8 (young pool) and from cells passaged 27-35 (senescent pool) were mixed (performed in quadruplicate).

2.2. Isolation and Characterization of Young and Senescent $e M V$. Young and senescent HUVEC-derived MVs were isolated from their culture medium. Briefly, samples were centrifuged using serial centrifugations $(15 \mathrm{~min}$ at $3000 \mathrm{rpm}$, $30 \mathrm{~min}$ at $14,000 \mathrm{rpm}$ ), and pellets were frozen and stored at $-20^{\circ} \mathrm{C}$ until use. Prior to use, MVs from cells passaged 4-8 (young pool) and from cells passaged 27-35 (senescent pool) were mixed (performed in quadruplicate).
MVs from a medium containing young and senescent HUVEC cells were characterized in terms of size using a Beckman Coulter Cytomics FC 500 flow cytometer running CXP software. MVs were considered to be those events gated with a size between $0.5-1.5 \mu \mathrm{m}$; this gate was established from the side scatter versus forward scatter dot plot produced in a standardization experiment using the SPHERO ${ }^{\mathrm{TM}}$ Flow Cytometry Nano Fluorescent Size Standard Kit (Spherotech). The latter has size-calibrated fluorescent beads ranging from $0.1-1.9 \mu \mathrm{m}$ in diameter. Events below $0.2 \mu \mathrm{m}$ were excluded in order to adequately distinguish true events from the background; events $>1.9 \mu \mathrm{m}$ were excluded to prevent possible confusion with apoptotic bodies. The absolute number of MVs (events) per $\mu \mathrm{L}$ was determined using Flow Count calibrator beads (Beckman Coulter) according to the manufacturer's recommendations and employing CXP software: (MVs counted $\times$ standard beads/L)/(standard beads counted). Data were recorded as the mean of three independent measurements of the same sample. The same number of senescent and young MVs was used in comparative analyses.

2.3. Western Blotting. The total protein content of extracts from young and senescent eMVs (performed using CytoBuster Protein Extraction Reagent lysis buffer (Millipore)), which contains a protease and a phosphatase inhibitor cocktail (Roche), was quantified using a BCA Protein Assay Kit (Pierce), employing BSA as the standard. Briefly, equal amounts of protein ( $25 \mu \mathrm{g}$ protein/lane) were diluted with a reducing sample buffer and separated by 4-20\% MiniPROTEAN ${ }^{\circledR}$ TGX $^{\mathrm{TM}}$ Precast Protein Gels under reducing conditions. These proteins were then transferred onto nitrocellulose membranes (BioRad), blocked with TBS containing $0.1 \%$ Tween 20 and 5\% dry nonfat milk for $1 \mathrm{~h}$ at room temperature, and incubated in the same buffer with different primary antibodies (anti-6PGL, Santa Cruz, sc-398833, dilution $1: 500,28 \mathrm{kDa}$; anti-GK, Santa Cruz, sc-393555, dilution 1/250, $61 \mathrm{kDa}$; anti-PSPH, Santa Cruz, sc-271421, dilution $1 / 250,25 \mathrm{kDa})$. After washing with TBST, the membranes were incubated with Novex horseradish peroxidaseconjugated secondary antibodies followed by 2 additional washing steps with TBST. Bands were visualized with Luminata Crescendo Western HRP substrate (Millipore). Ponceau red (Sigma) staining was used as a loading control. Bands were quantified using Image J software (NIH) and normalized to Ponceau red.

2.4. Mass Spectrometry for NADPH Analysis. The presence of $\mathrm{NADP}^{+}$and NADPH in eMVs was analyzed using a HPLC system Agilent 1100 coupled in-line to a TSQ Quantum triple quadrupole mass spectrometer (Thermo Scientific) equipped with an ESI source. Samples were prepared according to the "hot water/buffer extraction" protocol from Ortmayr [29]. Briefly, eMVs $\left(50 \times 10^{6}\right)$ were added to $250 \mu \mathrm{L}$ of $5 \mathrm{mM}$ ammonium acetate buffer at $\mathrm{pH}$ 8.0. The supernatant was collected after sample incubation for $3 \mathrm{~min}$ at $85^{\circ} \mathrm{C}$, cooling on dry ice, and centrifugation for $10 \mathrm{~min}$ at $4000 \times \mathrm{g}$. Equipment settings were also obtained from Ortmayr [29] (see Table 1). Mass spectra were recorded in negative mode for $\mathrm{NADP}^{+}$and NADPH. The column used was ACE Excel 
TABLE 1: Compound optimization table in MS/MS mode.

\begin{tabular}{lcc}
\hline & $\mathrm{NADP}^{+}$ & $\mathrm{NADPH}$ \\
\hline Molecular formula in MS & $\mathrm{C}_{21} \mathrm{H}_{27} \mathrm{~N}_{7} \mathrm{O}_{17} \mathrm{P}_{3}$ & $\mathrm{C}_{21} \mathrm{H}_{29} \mathrm{~N}_{7} \mathrm{O}_{17} \mathrm{P}_{3}$ \\
Parent mass & $742 \mathrm{~m} / \mathrm{z}$ & $744 \mathrm{~m} / \mathrm{z}$ \\
& $619.96 \mathrm{~m} / z / 17 \mathrm{v}$ & $426.16 \mathrm{~m} / z / 35 \mathrm{v}$ \\
Product ion $(\mathrm{m} / z) /$ collision & $407.89 \mathrm{~m} / z / 34 \mathrm{v}$ & $407.96 \mathrm{~m} / z / 36 \mathrm{v}$ \\
energy & $272.82 \mathrm{~m} / z / 38 \mathrm{v}$ & $396.96 \mathrm{~m} / \mathrm{z} / 32 \mathrm{v}$ \\
\hline
\end{tabular}

$3 \mathrm{C} 18$-PFP $150 \mathrm{~mm} \times 3.0 \mathrm{~mm}+3 \mu \mathrm{m}$. Mass spectra of the column eluates were recorded in MS/MS mode using methanol and $\mathrm{H}_{2} \mathrm{O}$, adding $5 \mathrm{mM}$ ammonium acetate. Nitrogen was used as the ion source gas. The sheath gas pressure was set at 40 (arbitrary units), the auxiliary gas pressure was set at 2 (arbitrary units), and the spray voltage was set at $3000 \mathrm{~V}$. The capillary temperature was set at $350^{\circ} \mathrm{C}$. Argon was used as the collision gas for collision-induced dissociation at a pressure of $1.5 \mathrm{mT}$ Torr (Q2). Data were acquired using Xcalibur Control Software.

2.5. Proteomic Analysis. Proteomic analysis involved in-gel protein digestion followed by HPLC and mass spectrometry (MS). In order to obtain sufficiently large samples, the four HUVEC “young pool” extracts (see Section 2.2) were mixed, as were the four HUVEC "senescent pool" extracts, and the corresponding pools for the MVs. These four samples were dissolved in lysis buffer (8 M urea, $2 \mathrm{M}$ thiourea, 5\% CHAPS, $2 \mathrm{mM}$ TCEP-HCl, and protease inhibitors). MVs were lysed by ultrasonication (10 strokes, low amplitude) on ice. The lysates were then centrifuged at $20,000 \times \mathrm{g}$ for $10 \mathrm{~min}$ at $4^{\circ} \mathrm{C}$, and the supernatant containing the solubilized proteins was used for LC-MS/MS experiments. Total protein concentration was determined using the Pierce $660 \mathrm{~nm}$ protein assay (Thermo). An aliquot of each sample was diluted with loading sample buffer and then loaded onto $1.2 \mathrm{~cm}$ wide wells of a conventional SDS-PAGE gel ( $1 \mathrm{~mm}$ thick, $4 \%$ stacking gel, and $12 \%$ resolving gel). The run was stopped as soon as the front entered $1 \mathrm{~cm}$ into the resolving gel, so that the whole proteome became concentrated at the stacking/resolving gel interface. The separated protein bands were visualized by Coomassie staining, excised, cut into cubes (cross section $1 \mathrm{~mm}^{2}$ ), deposited in 96-well plates, and processed automatically in a Proteineer DP (Bruker Daltonics). The digestion protocol used was based on Shevchenko et al. [30] with minor variations: gel plugs were washed firstly with $50 \mathrm{mM}$ ammonium bicarbonate and secondly with acetonitrile prior to reduction with $10 \mathrm{mM}$ dithiothreitol in $25 \mathrm{mM}$ ammonium bicarbonate solution; alkylation was performed with $55 \mathrm{mM}$ indoleacetic acid in $50 \mathrm{mM}$ ammonium bicarbonate solution. The gel pieces were then rinsed, firstly with $50 \mathrm{mM}$ ammonium bicarbonate and secondly with acetonitrile, and subsequently dried under a stream of nitrogen. Proteomics grade trypsin (Sigma-Aldrich) at a final concentration of $16 \mathrm{ng} / \mu \mathrm{L}$ in $25 \%$ acetonitrile/50 mM ammonium bicarbonate solution was added and digestion allowed to proceed at $37^{\circ} \mathrm{C}$ for $4 \mathrm{~h}$. The reaction was stopped by adding $50 \%$ acetonitrile $/ 0.5 \%$ trifluoroacetic acid for peptide extraction. The tryptic-eluted peptides were dried by speed- vacuum centrifugation and then desalted on StageTip C18 Pipette tips (Thermo Scientific) until analysis by mass spectrometry.

A $1 \mu \mathrm{g}$ aliquot of each sample was subjected to $1 \mathrm{D}$-nano LC ESI-MSMS analysis using an Eksigent Technologies nanoLC Ultra 1D plus nanoliquid chromatography system coupled to a high-speed Triple TOF 5600 mass spectrometer (SCIEX) with a Nanospray III source. The analytical column used was a silica-based reversed phase Acquity UPLC MClass Peptide BEH C18 Column $(75 \mu \mathrm{m} \times 150 \mathrm{~mm}, 1.7 \mu \mathrm{m}$ particle size, and $130 \AA$ pore size) (Waters). The trap column was a C18 Acclaim PepMap ${ }^{\text {TM }} 100$ (Thermo Scientific) $(100 \mu \mathrm{m} \times 2 \mathrm{~cm}, 5 \mu \mathrm{m}$ particle diameter, and $100 \AA$ pore size), switched on-line with the analytical column. The loading pump delivered a solution of $0.1 \%$ formic acid in water at $2 \mu \mathrm{L} / \mathrm{min}$. The nanopump provided a flow-rate of $250 \mathrm{~nL} /$ min and was operated under gradient elution conditions. Peptides were separated using a $2-90 \%$ mobile phase B gradient (mobile phase A: $2 \%$ acetonitrile, $0.1 \%$ formic acid; mobile phase B: $100 \%$ acetonitrile, $0.1 \%$ formic acid) for $250 \mathrm{~min}$. The injection volume was $5 \mu \mathrm{L}$.

Data acquisition was performed with a Triple TOF 5600 System (SCIEX) (ionspray voltage floating $2300 \mathrm{~V}$, curtain gas $35 \mathrm{psi}$, interface heater temperature $150^{\circ} \mathrm{C}$, ion source gas $125 \mathrm{psi}$, and declustering potential $100 \mathrm{~V}$ ). All data were acquired using information-dependent acquisition (IDA) mode with Analyst ${ }^{\circledR}$ TF 1.7 software (SCIEX). The following IDA parameters were chosen: a $0.25 \mathrm{~s}$ MS survey scan in the mass range $350-1250 \mathrm{Da}$, followed by $35 \mathrm{MS} / \mathrm{MS}$ scans of $100 \mathrm{~ms}$ in the mass range 100-1800 (total cycle time: $4 \mathrm{~s}$ ). Switching criteria were set to ions greater than a mass to charge ratio $(\mathrm{m} / \mathrm{z})$ of 350 and smaller than 1250 , with a charge state of 2-5 and an abundance threshold of more than 90 counts/s (cps). Former target ions were excluded for $15 \mathrm{~s}$. The IDA rolling collision energy (CE) parameters script was used for automatically controlling the CE.

MS and MS/MS data obtained from individual samples were processed using Analyst TF 1.7 software. Raw data file conversion tools were used to generate mgf files which were then compared (using Mascot Server v.2.5.1 software; Matrix Science) to those in the UniProt Homo sapiens protein database. The latter contains 40,530 coding genes and their corresponding reversed entries. The search parameters were set as follows: carbamidomethyl (C) as the fixed modification and acetyl (protein N-term) and oxidation (M) as the variable modifications. Peptide mass tolerance was set to $25 \mathrm{ppm}$ and $0.05 \mathrm{Da}$ for fragment mass. Two missed cleavages were allowed. False discovery rates ( $\leq 1 \%$ at the spectral level) for peptide identification were calculated manually.

2.6. Functional Experiments. Two functional experiments were carried out in order to (1) test the ability of MVs to use blood metabolites to feed the $\mathrm{NAD}(\mathrm{P}) \mathrm{H}$ synthesis machinery, and (2) visually demonstrate that these metabolites induce $\mathrm{NAD}(\mathrm{P}) \mathrm{H}$ formation.

In the first experiment, five different blood metabolites were tested separately on young and senescent eMVs: lactate, pyruvate, glucose, branched-chain amino acids (BCAA; Val, Leu, Ileu), and glycerol. The concentration used was higher 
than the plasma value found in the bibliography (included in Figure 1): $7 \mathrm{mM}, 0.5 \mathrm{mM}, 15 \mathrm{mM}, 0.3 \mathrm{mM}$, and $0.3 \mathrm{mM}$, respectively. In each tube, the test sample was formed by adding: $2 \mu \mathrm{L}$ of blood metabolite, $5 \mu \mathrm{L}$ of lysate including $10^{6}$ eMVs, and $5 \mu \mathrm{L}$ of $3 \mathrm{mM}$ NADPH (Santa Cruz Biotechnology, reference number 202725A). No metabolites were included in the control samples. NADPH was used as the internal control; in fact, this experimental design allowed us to analyze the metabolite-induced changes in the $\mathrm{NAD}(\mathrm{P})^{+} /$ $\mathrm{NAD}(\mathrm{P}) \mathrm{H}$ ratio. For example, for a certain metabolite, a lower ratio indicates that this metabolite increases the reduced form. After a $10 \mathrm{~min}$ incubation, $1 \mu \mathrm{L}$ of the sample was analyzed in a microvolume spectrophotometer (DeNovis DS-11 spectrophotometer), and the $\mathrm{NAD}(\mathrm{P})^{+} / \mathrm{NAD}(\mathrm{P}) \mathrm{H}$ ratio was determined by absorbance measurement at $260 \mathrm{~nm} / 340 \mathrm{~nm}$, respectively. The experiment was repeated three times using duplicated samples; twelve measures were performed in each case.

To visualize $\mathrm{NAD}(\mathrm{P}) \mathrm{H}$ synthesis, the eMVs (young and senescent) were incubated for $10 \mathrm{~min}$ with the blood metabolites previously described. Subsequently, $10 \mu \mathrm{L}$ of each sample including 40,000 eMVs was dropped into the center of a small water-repellent circle made on a slide with a PAP pen. The drop and the circle were wrapped up with a coverslip and observed in a Zeiss LSM 780 multiphoton confocal microscope with a Mai-Tai DS (690-1040 nm tunable) laser. The excitation wavelength was $735 \mathrm{~nm}$, more than twice that of $\mathrm{NAD}(\mathrm{P}) \mathrm{H}(340 \mathrm{~nm})$, and the laser intensity was set at $5.5 \%$. The detection range was 396-502 nm.

2.7. Statistical Analysis. Data were analyzed using $\mathrm{R}$ software. The two-tailed Student $t$-test was used to analyze differences in Western blotting results. Data of our first functional experiment (ability of MVs to use blood metabolites to feed the $\mathrm{NAD}(\mathrm{P}) \mathrm{H}$ synthesis machinery) were analyzed as follows. Kruskal-Wallis test (with post hoc Dunn's test) was performed for the comparison of the effect of metabolites in young eMVs and senescent eMVs. MannWhitney-Wilcoxon rank-sum test was used to compare the effect of every molecule between young and senescent: glucose in young versus glucose in senescent and so on. Significance was set at $p<0.05$.

\section{Results}

This section has been organized in three parts: (1) proteomic analysis, where we describe the biochemical pathways in eMVs which are directed at synthetizing NADPH; (2) the presence of $\mathrm{NADP}^{+}$in eMVs; and (3) functional analysis, to demonstrate whether or not eMVs can synthesize NAD $(\mathrm{P}) \mathrm{H}$ after activation of the pathways previously described. In the text that follows, the enzymes are named using the acronyms included in Table 2, the generic name (GN), and the metabolites with the acronyms included in Figure 1.

3.1. Proteomic Analysis. The pentose phosphate pathway (PPP) is the main biochemical route to synthesize NADPH. Hence, our first objective was to search for the presence of enzymes that participate in this pathway using mass spectrometry (MS) analysis of young and senescent eMVs. The majority of PPP enzymes were detected in this MS analysis, as shown in Table 2, except for 6PGL, GK, and PSPH, which were detected by Western blot (WB) analysis (Figure 2).

We next carried out a more detailed analysis of the enzymes detected in our MS results in order to connect the PPP with other biochemical routes. Table 2 includes the enzymes of the PPP and those of related metabolic routes proposed according to our proteomic analysis. To clarify these routes, a diagram of all the metabolic pathways proposed is included in Figure 1.

The organization of the different enzymes found in our proteomic analysis led us not only to establish the hypothetical biochemical routes that may be operative in the eMVs but also to point out the metabolites that feed these biochemical routes (Figure 1). All in all, it seems that both young and especially senescent eMVs have a very well-developed enzymatic organization designed to optimize NADPH production, and this is mainly due to the bidirectionality of most of these enzymatic activities. Besides the glycolytic pathways, which use glucose, lactate, and pyruvate, it is very important to point out the possibility that BCAA and glycerol can also be used to direct metabolites to the PPP. Mitochondria are also involved in this strategic metabolic organization: they can act as an NADPH biosynthetic organelle (note the high content of GLUD1 (number 22)) and supply AKG to the serine and 3PHP pathway. Enzymes involved in serine and glycerate metabolism are also included in these biochemical pathways using 3PHP and 3PG as metabolic intermediates.

One of the most interesting results was the higher content of most of these enzymes in senescent eMVs when compared with young eMVs; in fact, their enzymatic machinery seems to be designed to direct metabolites to the PPP or to obtain NADPH from new routes not included in young eMVs. The bold numbers in Table 2 indicate an increase $>25 \%$ when young and senescent eMVs were compared. A route specially used in senescent eMVs is the serine and PHP pathway, as suggested by the high content of the enzymes involved (numbers from 23 to 27 ) in senescent eMVs. The use of glutamine to form F6P using GFPT2 (number 28) is also especially elevated in senescent eMVs. Moreover, at the bottom of Table 2, we have included five enzymes that synthesize NADPH but are not included in Figure 1. Note that IDH1 and IDH2 are decreased in senescent eMVs, whereas the last three enzymes are increased.

3.2. Functional Analysis. We carried out two types of functional analysis; one to test the capacity of the proposed blood metabolites to feed the enzymatic machinery involved in reducing power synthesis and the other one to visualize this reducing power in eMVs. Unfortunately, NADH and NADPH are closely related molecules whose absorption and emission spectra are almost equal and, thereby, it has been impossible for us to separate $\mathrm{NADH}$ from $\mathrm{NADPH}$ in these functional analyses.

In order to study the effect of blood metabolites on $\mathrm{NAD}(\mathrm{P}) \mathrm{H}$ synthesis, eMVs were incubated $30 \mathrm{~min}$ with 


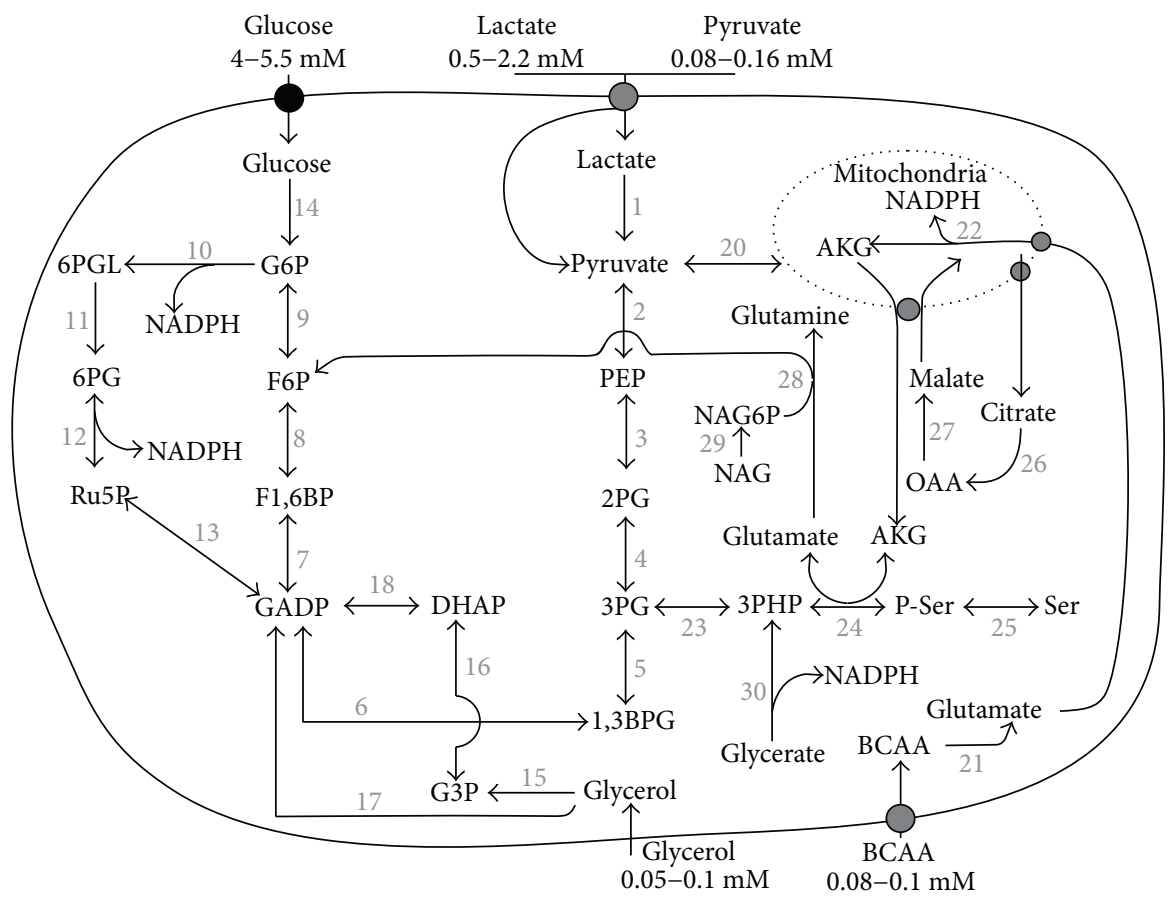

FIGURE 1: Schematic diagram of an eMV including the proposed metabolic pathways according to the proteomic results. The blood plasma metabolites that can feed these routes and their plasma concentrations under control conditions are also included. 1,3BPG: 1,3bisphosphoglycerate; 2PG: 2-phosphoglycerate; 3PG: 3-phosphoglycerate; 3PHP: 3-phosphohydroxypyruvate; 6PG: 6-phosphogluconate; 6PGL: 6-phosphoglucono-1,5-lactone; AKG: alpha-ketoglutarate; BCAA: branched-chain amino acids; DHAP: dihydroxyacetone 3-phosphate; F1,6BP: fructose 1,6-biphosphate; F6P: fructose 6-phosphate; G3P: glycerol 3-phosphate; G6P: glucose 6-phosphate; GADP: glyceraldehyde 3-phosphate; NAG: N-acetylglucosamine; NAG6P: N-acetylglucosamine-6-phosphate; OAA: oxaloacetate; PEP: phosphoenolpyruvate; P-Ser: phosphoserine; Ru5P: ribulose 5-phosphate.

the proposed metabolites (lactate, pyruvate, glucose, glycerol, and BCCA). These metabolites were incubated separately at a concentration that doubled the maximal value indicated in Figure 1. The absorbance ratio $260 \mathrm{~nm} /$ $340 \mathrm{~nm}$ is a measure of the relative proportion of $\mathrm{NAD}(\mathrm{P})^{+} / \mathrm{NAD}(\mathrm{P}) \mathrm{H}$. In young eMVs, lactate and pyruvate diminished significantly this ratio, which indicates that both metabolites induced the formation of $\mathrm{NAD}(\mathrm{P}) \mathrm{H}$. However, in senescent eMVs, all the metabolites tested induced a decrease in this ratio, glycerol and pyruvate being statistically significant. Statistically significant differences were also observed for glucose, glycerol, and BCAA when the effect of metabolites was compared in young and senescent eMVs (Figure 3).

An experiment using multiphoton confocal microscopy was performed to visualize the production of $\mathrm{NAD}(\mathrm{P}) \mathrm{H}$ by eMVs after incubation with the previously used metabolites. In order to detect only the reduced forms, eMVs were excited at $340 \mathrm{~nm}(670 \mathrm{~nm}$ in the multiphoton confocal microscope), and emission was detected at $460 \mathrm{~nm}$. Only after incubation with the proposed blood metabolites could eMVs be clearly visualized, demonstrating that eMVs have the capacity to synthesize reducing power $(\mathrm{NAD}(\mathrm{P}) \mathrm{H})$ (Figure 4), although not all vesicles were able to produce fluorescence. Only lactate and pyruvate induced fluorescence in young eMVs, and senescent eMVs seemed to be more efficient in producing fluorescence.
3.3. NADP ${ }^{+}$and NADPH in eMVs. An MS analysis was carried out to accurately detect the presence of $\mathrm{NADP}^{+}$and $\mathrm{NADPH}$ in eMVs. As can be seen in Figure 5, the presence of both cofactors was demonstrated in eMVs, although the content of $\mathrm{NADP}^{+}$was greater than that of the reduced form. Differences in the $\mathrm{NADP}^{+} / \mathrm{NADPH}$ ratio were not observed either following incubation with the blood metabolites or between young and senescent eMVs.

\section{Discussion}

In a recent work, we demonstrated the existence of a functional machinery for ROS detoxification in HUVEC eMVs [27]; now, our present study demonstrates that these HUVEC eMVs, especially those derived from senescent cells, can synthesize $\mathrm{NAD}(\mathrm{P}) \mathrm{H}$, the fuel that feeds the antioxidant machinery, used as precursors of different blood plasma metabolites. The combination of both results indicates that eMVs are subcellular structures with an autonomous capacity for ROS scavenging, as had been suggested by Soleti et al. [31]; eMV protection against oxidative stress regulating eNOS/Akt signaling has also been recently demonstrated [32]. Figure 6 shows a schematic drawing that represents our past and present results on the role of eMVs as ROS scavengers.

The presence of some antioxidant enzymes in plasma MVs [33-35], in endothelial-derived MVs [36], and in 
TABLE 2: Proteomic analysis of young and senescent eMVs. PS: protein score; PSM: peptide-spectrum match; NP: number of peptides (MS/ MS scores are sums for the validated peptides assigned to each protein); C: coverage. PS, PSM, and NP are usually considered quantitative variables in the proteomic analysis. Bold numbers in the "senescent columns" indicate a $25 \%$ increase in senescent eMVs; italic numbers in "young columns" indicate a $25 \%$ reduction in senescent eMVs. The 5 proteins at the bottom of the table are the enzymes that synthesize NADPH. UniProt: UniProt code; GN: generic name.

\begin{tabular}{|c|c|c|c|c|c|c|c|c|c|c|c|}
\hline \multirow{2}{*}{\multicolumn{2}{|c|}{ Enzymes }} & \multirow{2}{*}{ UniProt } & \multirow{2}{*}{ GN } & \multicolumn{4}{|c|}{ Young eMVs } & \multicolumn{4}{|c|}{ Senescent eMVs } \\
\hline & & & & PS & PSM & NP & $\mathrm{C} \%$ & PS & PSM & NP & $\mathrm{C} \%$ \\
\hline \multirow{4}{*}{1} & L-Lactate dehydrogenase B chain & P07195 & LDHB & 644 & 17 & 10 & 32 & 837 & 18 & 12 & 33.8 \\
\hline & L-Lactate dehydrogenase A chain & P00338 & LDHA & 458 & 14 & 7 & 25.6 & 1023 & 26 & 15 & 53.6 \\
\hline & L-Lactate dehydrogenase A-like 6A & Q6ZMR3 & LDHAL6A & 104 & 2 & 1 & 6.3 & 105 & 2 & 1 & 6.3 \\
\hline & L-Lactate dehydrogenase A-like 6B & Q9BYZ2 & LDHAL6B & & & & & 38 & 1 & 1 & 4.5 \\
\hline \multirow[t]{2}{*}{2} & Pyruvate kinase & P14618 & PKM & 1425 & 40 & 24 & 56.9 & 1675 & 44 & 24 & 60.8 \\
\hline & Alpha-enolase & P06733 & ENO1 & 810 & 14 & 10 & 49.8 & 1376 & 31 & 18 & 60.4 \\
\hline \multirow[t]{2}{*}{3} & Gamma-enolase & P09104 & ENO2 & 288 & 6 & 3 & 13.8 & 542 & 10 & 7 & 38.5 \\
\hline & Beta-enolase & P13929 & ENO3 & 319 & 6 & 3 & 13.6 & 425 & 9 & 5 & 18.7 \\
\hline 4 & Phosphoglycerate mutase 1 & P18669 & PGAM1 & 197 & 6 & 3 & 33.5 & 352 & 11 & 6 & 44.5 \\
\hline 5 & Phosphoglycerate kinase 1 & P00558 & PGK1 & 524 & 12 & 7 & 46.5 & 519 & 12 & 8 & 45.6 \\
\hline 6 & Glyceraldehyde-3-phosphate dehydrogenase & P04406 & GAPDH & 1645 & 50 & 23 & 75.5 & 1939 & 65 & 27 & 72.2 \\
\hline \multirow{2}{*}{7} & Fructose-bisphosphate aldolase A & P04075 & ALDOA & 565 & 12 & 9 & 44.2 & 1202 & 29 & 19 & 59.9 \\
\hline & Fructose-bisphosphate aldolase C & P09972 & ALDOC & 310 & 5 & 4 & 25.5 & 474 & 9 & 7 & 25.3 \\
\hline 8 & Fructose-2,6-bisphosphatase & Q9NQ88 & TIGAR & & & & & 46 & 1 & 1 & 6.7 \\
\hline 9 & Glucose-6-phosphate isomerase & P06744 & GPI & & & & & 272 & 6 & 5 & 20.6 \\
\hline 10 & Glucose-6-phosphate 1-dehydrogenase & P11413 & G6PD & & & & & 45 & 1 & 1 & 11.8 \\
\hline 11 & 6-Phosphogluconolactonase & O95336 & 6PGL & \multicolumn{8}{|c|}{ Western blot } \\
\hline 12 & 6-Phosphogluconate dehydrogenase, decarboxylating & P52209 & PGD & 162 & 3 & 3 & 22.2 & 160 & 3 & 3 & 24.2 \\
\hline 13 & Transketolase & P29401 & TKT & 238 & 7 & 4 & 14.4 & 185 & 6 & 4 & 19.3 \\
\hline 14 & Hexokinase-1 & P19367 & HK1 & 265 & 5 & 5 & 16.4 & 262 & 7 & 5 & 9.6 \\
\hline 15 & Glycerol kinase & P32189 & GK & \multicolumn{8}{|c|}{ Western blot } \\
\hline 16 & Glycerol-3-phosphate dehydrogenase, mitochondrial & P43304 & GPD2 & 52 & 1 & 1 & 39 & & & & \\
\hline 17 & Alcohol dehydrogenase [NADP(+)] & P14550 & AKR1A1 & & & & & 44 & 1 & 1 & 7.1 \\
\hline 18 & Triosephosphate isomerase & P60174 & TPI1 & 434 & 8 & 7 & 52.4 & 523 & 12 & 8 & 54.9 \\
\hline \multirow{2}{*}{19} & Glutamine-fructose-6-phosphate aminotransferase & O94808 & GFPT2 & 43 & 1 & 1 & 4.3 & 84 & 4 & 2 & 4.4 \\
\hline & Glutamine-fructose-6-phosphate aminotransferase & Q06210 & GFPT1 & & & & & 241 & 4 & 4 & 8.9 \\
\hline \multirow{2}{*}{20} & Pyruvate dehydrogenase E1 component subunit alpha & P08559 & PDHA1 & 5 & 3 & 13.8 & 80 & 2 & 1 & 5.4 & \\
\hline & Pyruvate dehydrogenase E1 component subunit beta & P11177 & PDHB & 114 & 2 & 2 & 17.3 & 38 & 1 & 1 & 9.7 \\
\hline 21 & Branched-chain amino acid aminotransferase, mitochondria & 015382 & BCAT2 & & & & & 94 & 1 & 1 & 6.4 \\
\hline 22 & Glutamate dehydrogenase 1 , mitochondrial & P00367 & GLUD1 & 329.0 & 11.0 & 5.0 & 24.0 & 637.0 & 17.0 & 11.0 & 35.5 \\
\hline 23 & D-3-Phosphoglycerate dehydrogenase & 043175 & PHGDH & 80 & 2 & 1 & 5.1 & 391 & 8 & 6 & 23.3 \\
\hline 24 & Phosphoserine aminotransferase & Q9Y617 & PSAT1 & & & & & 47 & 1 & 1 & 5.4 \\
\hline 25 & Phosphoserine phosphatase & PSPH & P78330 & \multicolumn{8}{|c|}{ Western blot } \\
\hline 26 & ATP-citrate synthase & P53396 & ACLY & 286 & 6 & 5 & 16.7 & 358 & 8 & 6 & 13.4 \\
\hline 27 & Malate dehydrogenase, cytoplasmic & P40925 & MDH1 & 99 & 3 & 1 & 10.8 & 176 & 5 & 3 & 17.7 \\
\hline 28 & Glutamine-fructose-6-phosphate aminotransferase & O94808 & GFPT2 & 43 & 1 & 1 & 4.3 & 84 & 4 & 2 & 4.4 \\
\hline 29 & N-Acetyl-D-glucosamine kinase & Q9UJ70 & NAGK & & & & & 193 & 3 & 3 & 22.1 \\
\hline 30 & Glyoxylate reductase/hydroxypyruvate reductase & Q9UBQ7 & GRHPR & & & & & 38 & 1 & 1 & 3 \\
\hline \multicolumn{12}{|c|}{ Other NAD(P)H-related enzymes } \\
\hline & Isocitrate dehydrogenase (NADP) cytoplasmic & O75874 & IDH1 & 41 & 1 & 1 & 2.7 & & & & \\
\hline & Isocitrate dehydrogenase (NADP), mitochondrial & P48735 & IDH2 & 254.0 & 6.0 & 5.0 & 38.7 & 133.0 & 3.0 & 3.0 & 30.8 \\
\hline & $\begin{array}{c}\text { Flavin reductase (NADPH) Alpha-aminoadipic } \\
\text { semialdehyde }\end{array}$ & P30043 & BLVRB & & & & & 37 & 1 & 1 & 12.1 \\
\hline & Alpha-aminoadipic semialdehyde dehydrogenase & P49419 & ALDH7A1 & 111 & 2 & 2 & 11.9 & 207 & 5 & 4 & 16.5 \\
\hline & $\mathrm{NAD}(\mathrm{P}) \mathrm{H}$ dehydrogenase (quinone) & P15559 & NQO1 & & & & & 39 & 1 & 1 & 6.6 \\
\hline
\end{tabular}




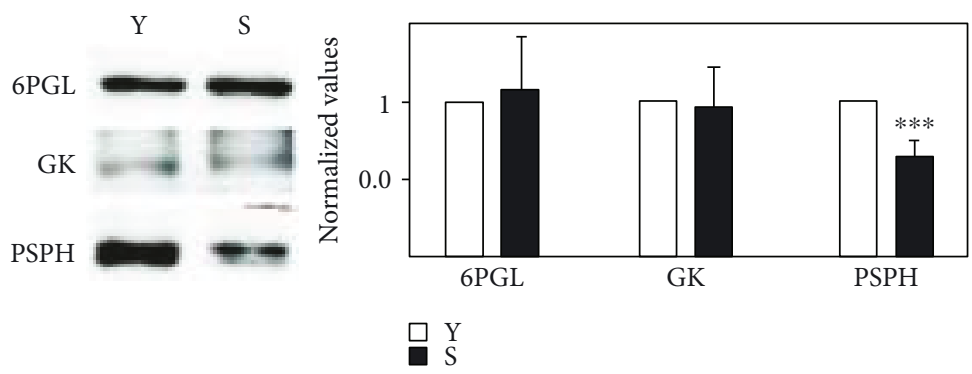

FIGURE 2: Western blot analysis of 6PGL, GK, and PSPH and the corresponding normalized analysis. A representative pool is included in the figure: young (Y) and senescent (S). THP-1 cells were used as a positive control (not shown). Bands were located at the expected molecular weight of 6PGL $(28 \mathrm{kDa})$, GK $(61 \mathrm{kDa})$, and PSPH $(25 \mathrm{kDa})$. Protein data for the eMVs was normalized against the intensity of Ponceau red staining. Bars represent mean $\pm \mathrm{SD}(n=4$ pools $) .{ }^{* * *} p<0.001$.
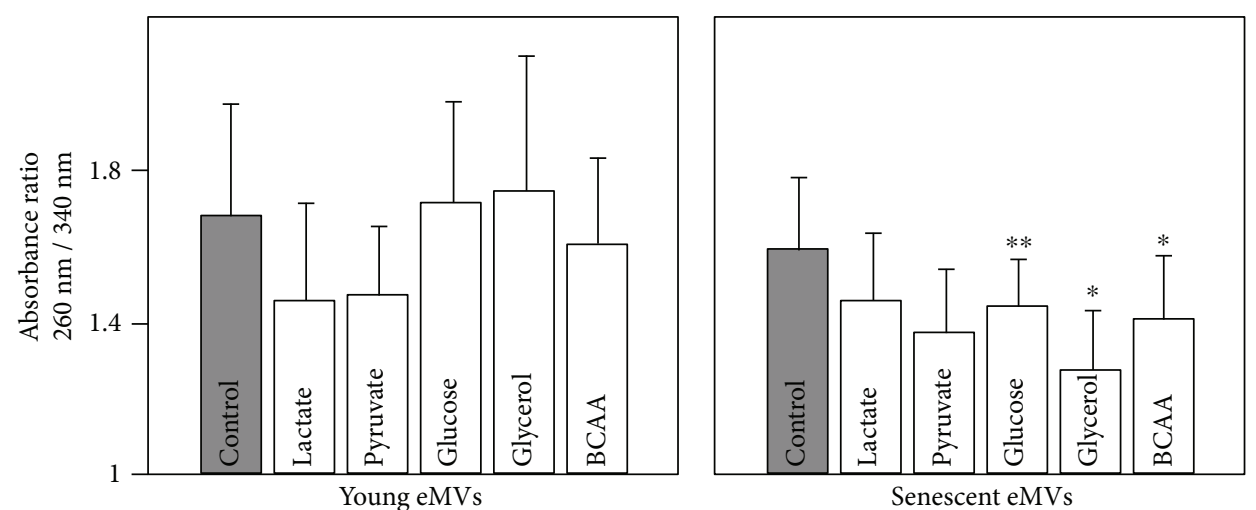

FIGURE 3: Effect of incubating eMVs with different blood plasma metabolites on the ratio of absorbance at $260 \mathrm{~nm}$ and $340 \mathrm{~nm}$, absorption peaks of $\mathrm{NAD}(\mathrm{P})$ and $\mathrm{NAD}(\mathrm{P}) \mathrm{H}$, respectively. No blood metabolites were added to control eMVs. Error bars represent SD; $n=3$. To clarify the plot, only statistical significance of the Mann-Whitney-Wilcoxon rank-sum test is included. ${ }^{*} p<0.05,{ }^{* *} p<0.01$. In the Kruskall-Wallis test for young eMVs, significant differences were observed in control versus lactate and pyruvate $(p<0.05)$ and between glucose versus lactate $(p<0.01)$. Significant differences were also observed for senescent eMVs in control versus glycerol and pyruvate $(p<0.05)$ and between glycerol versus glucose and lactate $(p<0.01)$.

HUVEC-derived MVs [31] had been already demonstrated. However, in our previous work [27], we demonstrated that HUVECs had a complete antioxidant machinery and that their MVs included a specific group of functional enzymes mainly involved in $\mathrm{O}_{2}^{-}$and $\mathrm{H}_{2} \mathrm{O}_{2}$ detoxification. Moreover, to date, the possibility of NADPH synthesis in MVs has not been considered in the literature. As far as we know, this is the first time that the capacity of NADPH synthesis is ascribed to eMVs, although the existence of some PPP enzymes has been demonstrated in exosomes [37], and some enzymes included in Table 2 have also been found in different proteomic studies: numbers $1,5,6,7$, and 18 and IDH2 [36]; numbers 5, 6, and 7 [33]; numbers 6 and 7 and ALDH [35]; and numbers 10, 20, and 27 and ALDH [34]. The synthesis of NADPH in eMVs seems logical; it makes no sense to contain an antioxidant machinery without the capacity of synthesizing the molecule that feeds it.

NADPH is considered the essential reductant for antioxidant systems. Unfortunately, its absorption and emission spectra are very similar to that of NADH. The appearance of FLIM [38, 39] and MS [29] methods, however, has made it possible to study these metabolites separately. FLIM permits the monitoring of MVs, but the use of MS is ideal for quantitative comparative studies of oxidized $\left(\mathrm{NADP}^{+}\right)$ and reduced (NADPH) forms. eMVs have an important handicap: it is difficult and very expensive to obtain large amounts of eMVs using HUVEC. This handicap has made our attempts to determine the $\mathrm{NADP}^{+} / \mathrm{NADPH}$ ratio using the standard commercial kits for NADPH analysis impossible. This problem can be resolved using FLIM, but this technique is very expensive and not usually available in most laboratories. While it is true that MS does not require big amounts of sample, even so, it is complicated to obtain enough eMVs for MS, and this problem gets worse in endothelial senescent cells because it is not easy to reach PD $>96$. Note that the volume of $50 \times 10^{6} \mathrm{eMVs}$ can be, more or less, $0.025 \mathrm{~mm}^{3}$ of sample. Moreover, NADPH is not very stable [40]; heat specially induces its oxidation to $\mathrm{NADP}^{+}$, and MS samples have to be intensely heated up. This might explain not only the low signal of NADPH but also the lack of differences observed in our MS analysis after incubation with blood metabolites or between young and senescent eMVs. In addition, NADPH has a lower-one order of magnitude-response factor than $\mathrm{NADP}^{+}$. The filtering and 


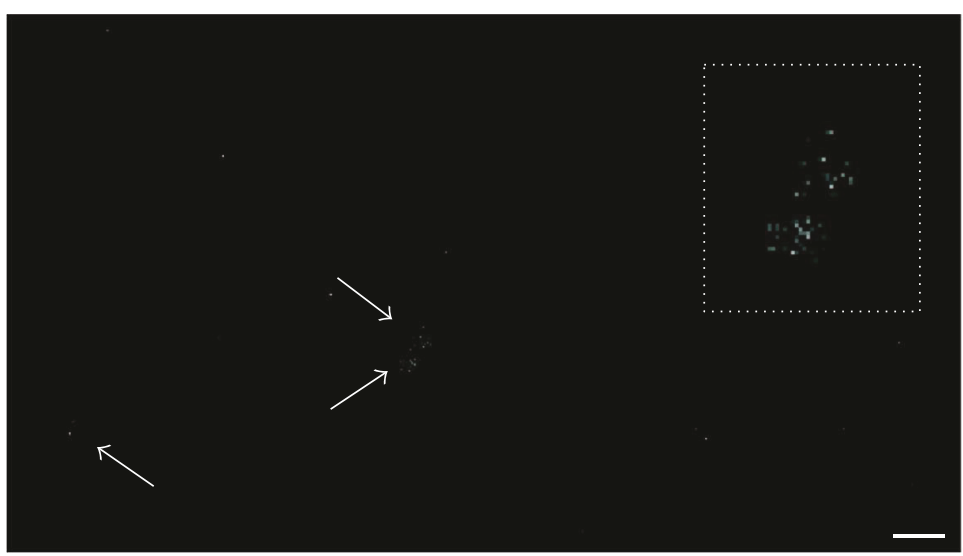

(a)

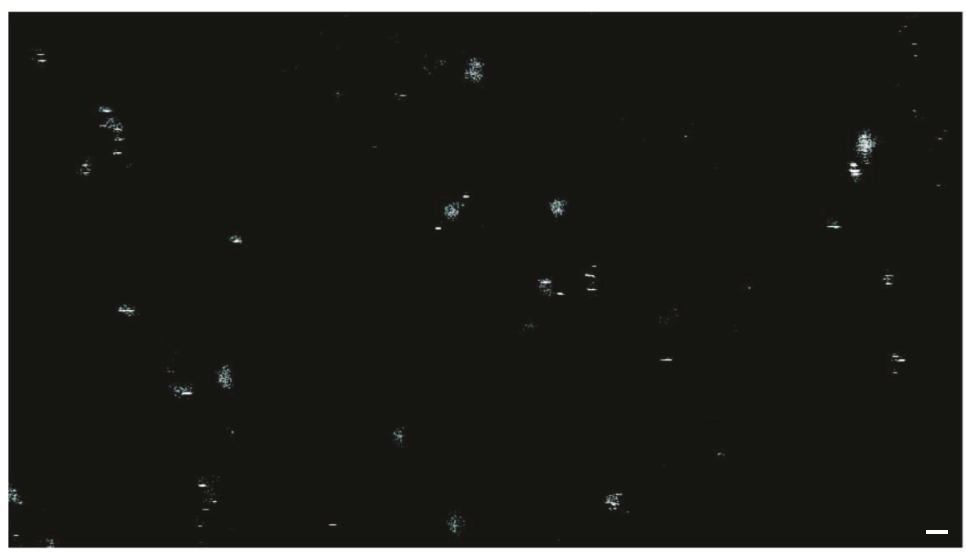

(b)

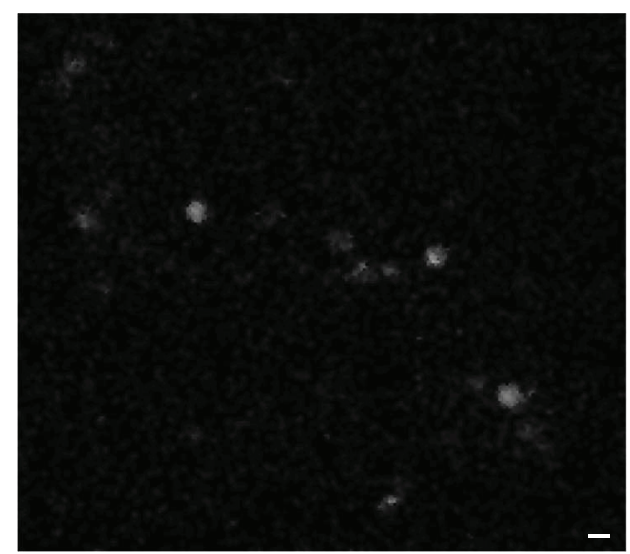

(c)

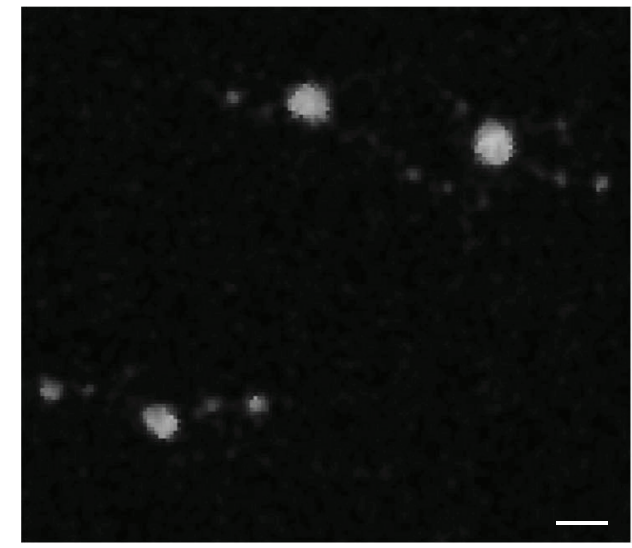

(d)

FIGURE 4: eMVs observed with a multiphoton confocal microscope. (a) Young eMVs under control conditions; arrows indicate eMVs. The square delimited by a dotted line is a magnification of the two eMVs indicated by the two arrows. (b) Senescent eMVs after glycerol incubation. (c) Young eMVs after lactate incubation. (d) Senescent eMVs after pyruvate incubation. The different size of the eMVs in the image is due to the fact that the eMVs were floating in the buffer in different positions in the $z$-axis. eMVs were obtained after mixing four pools. Scale bar, $1 \mu \mathrm{m}$.

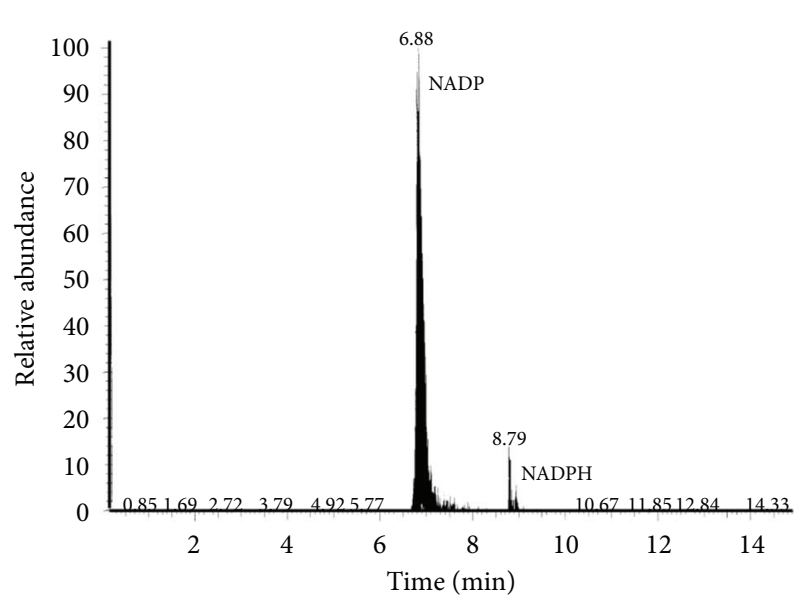

FIgURE 5: MS analysis of $\mathrm{NADP}^{+}$(6.88 elution time) and NADPH (8.79 elution time) content in senescent eMVs. eMVs were obtained after mixing four pools. $\mathrm{NADP}^{+} \mathrm{MW}$ : $742 \mathrm{D}, \mathrm{NADPH}$ MW: 744 D. dilution of the samples, as well as the existence of carbohydrates in the molecule (the ribose rings), that makes MS analysis difficult, must also be considered.

In this work, we propose a model of organization of the metabolic routes in eMVs in accordance with our proteomic analysis (MS and WB) (see Figure 1). Although both young and senescent eMVs can synthesize reducing power, the latter have a stronger synthetic machinery. In fact, it seems that senescent eMVs redesign their metabolic machinery to optimize NADPH synthesis. This new metabolic reorganization has some interesting hallmarks. Mitochondrial activity is deeply affected in order to diminish its activity: (1) ADP/ ATP translocases diminish or disappear: translocase 1 from 362 (young eMVs) to 0 (senescent eMVs) (protein score values), translocase 2 from 580 to 102, and translocase 3 from 549 to 0 ; (2) mitochondrial aminotransferases also diminish or disappear: ornithine aminotransferase from 55 to 0 , aspartate aminotransferase from 520 to 251, and serine hydroxymethyltransferase from 339 to 122; (3) pyruvate dehydrogenase which contributes to transforming pyruvate into acetyl-CoA, a metabolite used in the tricarboxylic acid 


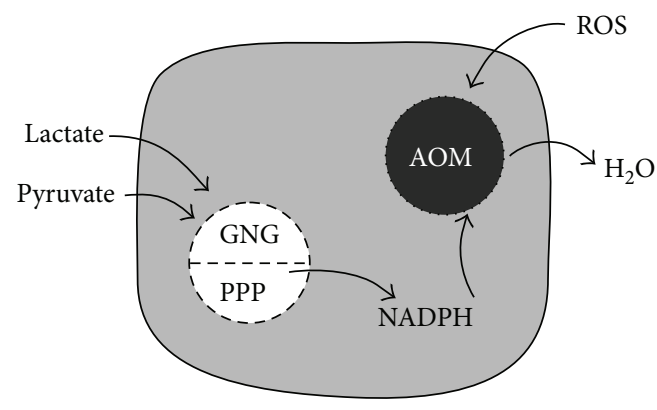

(a)

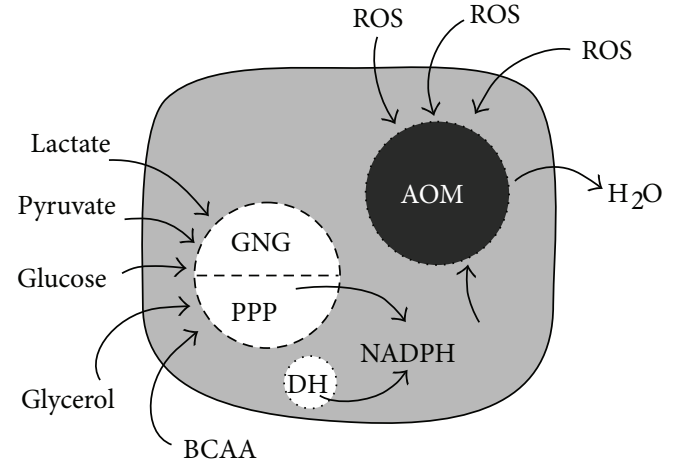

(b)

FIGURE 6: A young (a) and a senescent (b) eMV. AOM: antioxidant machinery; PPP: pentose phosphate pathway; GNG: gluconeogenesis; ROS: reactive oxygen species; DH: dehydrogenases; BCAA: branched-chain amino acids. Note that the senescent eMV has larger enzymatic machineries, uses more metabolites to feed them, and also has a higher capacity for ROS scavenging.

(TCA) cycle, is diminished in senescent eMVs (see enzyme number 20 in Table 2); and (4) the TCA enzymes are unbalanced; for example, succinic dehydrogenase is not present in senescent eMVs, whereas isocitrate dehydrogenase and aconitase are diminished. The incorporation of pyruvate to the glycolytic pathway (note that PK (number 2 ) is augmented) and the sequestering of AKG in the serine and glutamine pathways in order to synthesize 3PG and F6P are in accordance with this depletion of mitochondrial metabolic activity.

It is well known that the main enzymes involved in NADPH synthesis are PPP dehydrogenases [41], isocitrate dehydrogenases, aldehyde dehydrogenases, malic enzyme, and NAD kinase [42]. PPP dehydrogenases (enzyme numbers 10 and 12) and alcohol (enzyme number 17) and aldehyde dehydrogenases are increased in senescent eMVs, as well as $\mathrm{NAD}(\mathrm{P}) \mathrm{H}$ dehydrogenase and flavin reductase (see the last three enzymes of Table 2). Moreover, two other dehydrogenases, GLUD1 (number 22), involved in mitochondrial NADPH synthesis, and LDH (number 1) (NAD-dependent), are strongly increased. Isocitrate dehydrogenase, however, is diminished (see above paragraph), and malic enzyme and NAD kinase have not been detected. A serine-related folatedependent NADPH production has been demonstrated in proliferating [43] and in cancer [44] cells; however, the enzymes of this route have not been detected in our study.

Under high oxidative conditions, the PPP consumes more glucose to compensate for the depleted GSH [45]; in fact, a high oxidative stress drives G6P to the PPP, generating NADPH for antioxidant defenses [46], and a mechanistic link between increased G6PD (number 10) activity, elevated $\mathrm{NADPH}$, and improved antioxidant protection has been suggested [47]. An activation of the PPP has also been demonstrated in cancer cells, probably to produce more NADPH to combat oxidative stress [48]; indeed, the existence of PPP enzymes has been demonstrated in exosomes of ovarian cancer cells in proteomic analysis [37]. Human skin cells also activate the PPP in response to oxidative stress [49], and senescent fibroblasts show increased glycolysis and PPP to reduce ROS production, upregulation of pathways involved in redox homeostasis [50], and alterations in nicotinamide metabolism [51]. Moreover, another different metabolic possibility has been suggested for ROS scavenging; the enzyme biliverdin reductase has been involved in a ROS-scavenging mechanism in a subpopulation of epididymal fluid MVs, protecting spermatozoa against ROS released from dying cells [52].

Evidence that MVs are a heterogeneous population is continuously increasing [53]. This heterogeneity can be considered at least from two different points of view: structural (size) and functional (biochemical). In our functional analysis using multiphoton confocal microscopy, not all MVs emitted fluorescence after their stimulation. In fact, we think that only a part of the eMV s could act as ROS scavengers. We have just seen that to accomplish this function, it is necessary to reorganize the metabolic routes of the eMVs, and it is logical to assume that this drastic reorganization may not be useful for other functions supported by MVs; for example, for those MVs capable of ROS synthesis and involved in signaling processes [54-56].

eMVs, or at least a subpopulation of them, produce reducing power, but the enzymatic machinery involved in this process also needs to be fed. In this study, we have demonstrated that lactate, pyruvate, glucose, glycerol, and BCAA fuel this reducing power synthetic machinery. All these metabolites are present in blood plasma and, except glycerol that diffuses freely, need membrane transporters to cross the plasma membrane and get into the MVs. Lactic acid can also diffuse freely; however, in the blood, it is dissociated and present as lactate (ionized) that cannot pass through the plasma membrane. The use of these metabolites is different in young (only use lactate and pyruvate) and senescent eMVs (use all). Obviously, the higher reducing power synthesis capacity of senescent cells is in accordance with their wider metabolite availability. On the other hand, the use of some of these metabolites could represent another metabolic advantage; for example, the use of lactate may serve to regulate lactate concentration and to prevent lactic acidosis and redox regulation in plasma. We have not detected monocarboxylate transporters (lactate and pyruvate transporters), neutral amino acid transporters, or glucose transporters in our proteomic study; however, the fluorescence emission 
after incubation of the eMVs with these metabolites implies the existence of a transport system. A low expression of these transporters could explain this result, but the fast (a few minutes) emission of fluorescence in eMVs after incubation with these metabolites suggests the contrary. The possibility that the membrane proteins, due to their hydrophobicity, are harder to detect in MS, indeed requiring thiourea to solubilize them, should be considered.

The contribution of ROS to aging has been widely documented, although the beneficial effects produced by ROS have led to a new interpretation of this traditional view [57-59]. An increase of ROS production has also been related with senescence, an experimental model widely used in aging studies $[20,24,60]$ and also demonstrated in our previous work in senescent endothelial cells [27]. In accordance with the stronger capacity of ROS elimination demonstrated by senescent eMVs in our previous work, the present study demonstrates a strong increase of the machinery involved in NADPH synthesis in senescent eMVs. However, the stronger capacity of senescent endothelial cells and their MVs to eliminate ROS and the higher ROS production observed in senescence seems paradoxical. Their increased capacity for ROS scavenging might be an adaptive mechanism to the higher oxidative stress of senescent cells, and it could be considered a strategy used by senescent cells to promote cell survival. Unfortunately, this increased ROS scavenging is unable to compensate the higher levels of oxidative stress associated with senescence. On the other hand, eMVs can not only eliminate ROS but can also be effective in protecting against oxidative stress, suppressing NADPH oxidase and ROS production [32]. Finally, it should be considered that these results are obtained using the in vitro replicative senescent model in HUVEC; undoubtedly, this is a starting point that needs to be confirmed in in vivo aging models.

\section{Conclusions}

Our present and previous results demonstrate that eMVs can act as autonomous ROS scavengers: they use blood metabolites to synthesize NADPH that fuels their antioxidant machinery; moreover, senescent eMVs have a stronger ROS-scavenging capacity. Whether alterations in the ability of eMVs to regulate the oxidant/antioxidant balance can act as etiopathogenic mechanisms in diseases associated to a misbalance in oxidative stress is left out of this study but probably merits to be considered in order to use these MVs as diagnostic tools and/or therapeutic targets.

\section{Conflicts of Interest}

The authors have no conflicts of interest to declare.

\section{Authors' Contributions}

Guillermo Bodega, Matilde Alique, and Manuel R. Ramírez designed the study. Sergio Ciordia and María C. Mena carried out mass spectrometry analysis; Matilde Alique the Western blot analysis; Guillermo Bodega, Lourdes Bohórquez, Miriam Morán, and Luis Magro the functional analysis; and Guillermo
Bodega and Elvira Arza the multiphoton confocal microscopy. Guillermo Bodega, Matilde Alique, Lilian Puebla, and Manuel R. Ramírez wrote the manuscript. All authors read and approved the final manuscript.

\section{Acknowledgments}

This work was funded by the Plan Nacional Proyectos de Investigación en Salud of Instituto de Salud Carlos III (ISCIII), EU FEDER Grant (PI14/00806). All proteomic analyses were performed at the Proteomics Facility of the Spanish National Center for Biotechnology (CNB-CSIC) (a member of ProteoRed, PRB2-ISCIII, financed via grant PT13/0001). The authors thank José María Arribas (Espectrometría de Masas y Análisis Elemental, Universidad de Alcalá) for NADPH analysis and Dr. Marcos Marvá (Departamento de Física y Matemáticas, Universidad de Alcalá) for statistical analysis.

\section{References}

[1] E. van der Pol, A. N. Böing, P. Harrison, A. Sturk, and R. Nieuwland, "Classification, functions, and clinical relevance of extracellular vesicles," Pharmacological Reviews, vol. 64, no. 3, pp. 676-705, 2012.

[2] M. Colombo, G. Raposo, and C. Théry, "Biogenesis, secretion, and intercellular interactions of exosomes and other extracellular vesicles," Annual Review of Cell and Developmental Biology, vol. 30, no. 1, pp. 255-289, 2014.

[3] M. Yáñez-Mó, P. R. M. Siljander, Z. Andreu et al., "Biological properties of extracellular vesicles and their physiological functions," Journal of Extracellular Vesicles, vol. 4, no. 1, article 27066, 2015.

[4] J.-D. Tissot, G. Canellini, O. Rubin et al., "Blood microvesicles: from proteomics to physiology," Translational Proteomics, vol. 1, no. 1, pp. 38-52, 2013.

[5] S. M. Craige, S. Kant, and J. F. Keaney Jr., "Reactive oxygen species in endothelial function - from disease to adaptation," Circulation Journal, vol. 79, no. 6, pp. 1145-1155, 2015.

[6] M. L. Liu and K. J. Williams, "Microvesicles: potential markers and mediators of endothelial dysfunction," Current Opinion in Endocrinology, Diabetes and Obesity, vol. 19, no. 2, pp. 121127, 2012.

[7] A. Fleury, M. C. Martinez, and S. Le Lay, "Extracellular vesicles as therapeutic tools in cardiovascular diseases," Frontiers in Immunology, vol. 5, p. 370, 2014.

[8] D. Vara and G. Pula, "Reactive oxygen species: physiological roles in the regulation of vascular cells," Current Molecular Medicine, vol. 14, no. 9, pp. 1103-1125, 2014.

[9] Y. Zhou, H. Yan, M. Guo, J. Zhu, Q. Xiao, and L. Zhang, "Reactive oxygen species in vascular formation and development," Oxidative Medicine and Cellular Longevity, vol. 2013, Article ID 374963, 14 pages, 2013.

[10] M. El Assar, J. Angulo, S. Vallejo, C. Peiró, C. F. SánchezFerrer, and L. Rodríguez-Mañas, "Mechanisms involved in the aging-induced vascular dysfunction," Frontiers in Physiology, vol. 3, p. 132, 2012.

[11] M. El Assar, J. Angulo, and L. Rodríguez-Mañas, "Oxidative stress and vascular inflammation in aging," Free Radical Biology \& Medicine, vol. 65, pp. 380-401, 2013. 
[12] R. Brandes, I. Fleming, and R. Busse, "Endothelial aging," Cardiovascular Research, vol. 66, no. 2, pp. 286-294, 2005.

[13] Y. Mikhed, A. Daiber, and S. Steven, "Mitochondrial oxidative stress, mitochondrial DNA damage and their role in agerelated vascular dysfunction," International Journal of Molecular Sciences, vol. 16, no. 12, pp. 15918-15953, 2015.

[14] A. A. Puca, A. Carrizzo, F. Villa et al., "Vascular ageing: the role of oxidative stress," The International Journal of Biochemistry \& Cell Biology, vol. 45, no. 3, pp. 556-559, 2013.

[15] N. S. Dhalla, R. M. Temsah, and T. Netticadan, "Role of oxidative stress in cardiovascular diseases," Journal of Hypertension, vol. 18, no. 6, pp. 655-673, 2000.

[16] H. Li, S. Horke, and U. Förstermann, "Oxidative stress in vascular disease and its pharmacological prevention," Trends in Pharmacological Sciences, vol. 34, no. 6, pp. 313-319, 2013.

[17] N. R. Madamanchi, A. Vendrov, and M. S. Runge, "Oxidative stress and vascular disease," Arteriosclerosis, Thrombosis, and Vascular Biology, vol. 25, pp. 29-38, 2005.

[18] D. J. Kurz, S. Decary, Y. Hong, E. Trivier, A. Akhmedov, and J. D. Erusalimsky, "Chronic oxidative stress compromises telomere integrity and accelerates the onset of senescence in human endothelial cells," Journal of Cell Science, vol. 117, no. 11, pp. 2417-2426, 2004.

[19] E. Schulz, E. Anter, and J. F. Keaney Jr., "Oxidative stress, antioxidants, and endothelial function," Current Medicinal Chemistry, vol. 11, no. 9, pp. 1093-1104, 2004.

[20] J. D. Erusalimsky and C. Skene, "Mechanisms of endothelial senescence," Experimental Physiology, vol. 94, no. 3, pp. 299304, 2009.

[21] V. Victor, N. Apostolova, R. Herance, A. Hernandez-Mijares, and M. Rocha, "Oxidative stress and mitochondrial dysfunction in atherosclerosis: mitochondria-targeted antioxidants as potential therapy," Current Medicinal Chemistry, vol. 16, no. 35, pp. 4654-4667, 2009.

[22] D. R. Seals, K. L. Jablonski, and A. J. Donato, "Aging and vascular endothelial function in humans," Clinical Science, vol. 120, no. 9, pp. 357-375, 2011.

[23] A. J. Donato, R. G. Morgan, A. E. Walker, and L. A. Lesniewski, "Cellular and molecular biology of aging endothelial cells," Journal of Molecular and Cellular Cardiology, vol. 89, Part B, pp. 122-135, 2015.

[24] R. Liu, H. Liu, Y. Ha, R. G. Tilton, and W. Zhang, “Oxidative stress induces endothelial cell senescence via downregulation of Sirt6," BioMed Research International, vol. 2014, Article ID 902842, 13 pages, 2014.

[25] W. Ying, "NAD ${ }^{+} / \mathrm{NADH}$ and $\mathrm{NADP}^{+} / \mathrm{NADPH}$ in cellular functions and cell death: regulation and biological consequences," Antioxidants \& Redox Signaling, vol. 10, no. 2, pp. 179-206, 2008.

[26] L. Agledal, M. Niere, and M. Ziegler, "The phosphate makes a difference: cellular functions of NADP," Redox Report, vol. 15, no. 1, pp. 2-10, 2010.

[27] G. Bodega, M. Alique, L. Bohórquez, S. Ciordia, M. C. Mena, and M. R. Ramírez, "The antioxidant machinery of young and senescent human umbilical vein endothelial cells and their microvesicles," Oxidative Medicine and Cellular Longevity, vol. 2017, Article ID 7094781, 12 pages, 2017.

[28] J. Carracedo, P. Buendía, A. Merino et al., "Cellular senescence determines endothelial cell damage induced by uremia," Experimental Gerontology, vol. 48, no. 8, pp. 766-773, 2013.
[29] K. Ortmayr, Quantitative Analysis of NADP ${ }^{+}$and NADPH in Yeast, [M.S. thesis], University of Natural Resources and Life Science, Vienna, Austria, 2013.

[30] A. Shevchenko, M. Wilm, O. Vorm, and M. Mann, "Mass spectrometric sequencing of proteins silver-stained polyacrylamide gels," Analytical Chemistry, vol. 68, no. 5, pp. 850858, 1996.

[31] R. Soleti, E. Lauret, R. Andriantsitohaina, and M. Carmen Martínez, "Internalization and induction of antioxidant messages by microvesicles contribute to the antiapoptotic effects on human endothelial cells," Free Radical Biology \& Medicine, vol. 53, no. 11, pp. 2159-2170, 2012.

[32] A. M. Mahmoud, F. L. Wilkinson, E. M. McCarthy et al., "Endothelial microparticles prevent lipid-induced endothelial damage via Akt/eNOS signaling and reduced oxidative stress," The FASEB Journal, vol. 31, no. 10, pp. 4636-4648, 2017.

[33] M. Jin, G. Drwal, T. Bourgeois, J. Saltz, and H. M. Wu, "Distinct proteome features of plasma microparticles," Proteomics, vol. 5, no. 7, pp. 1940-1952, 2005.

[34] D. B. Peterson, T. Sander, S. Kaul et al., "Comparative proteomic analysis of PAI-1 and TNF-alpha-derived endothelial microparticles," Proteomics, vol. 8, no. 12, pp. 2430-2446, 2008.

[35] D. Povero, A. Eguchi, H. Li et al., "Circulating extracellular vesicles with specific proteome and liver microRNAs are potential biomarkers for liver injury in experimental fatty liver disease," PLoS One, vol. 9, no. 12, article e113651, 2014.

[36] K. Little, D. Smalley, N. Harthun, and K. Ley, "The plasma microparticle proteome," Seminars in Thrombosis and Hemostasis, vol. 36, no. 8, pp. 845-856, 2010.

[37] H. Yi, X. Zheng, J. Song, R. Shen, Y. Su, and D. Lin, "Exosomes mediated pentose phosphate pathway in ovarian cancer metastasis: a proteomics analysis," International Journal of Clinical and Experimental Pathology, vol. 8, no. 12, pp. 15719-15728, 2015.

[38] T. S. Blacker, Z. F. Mann, J. E. Gale et al., "Separating NADH and NADPH fluorescence in live cells and tissues using FLIM," Nature Communications, vol. 5, p. 3936, 2014.

[39] T. S. Blacker and M. R. Duchen, "Investigating mitochondrial redox state using NADH and NADPH autofluorescence," Free Radical Biology \& Medicine, vol. 100, pp. 53-65, 2016.

[40] J. T. Wu, L. H. Wu, and J. A. Knight, "Stability of NADPH: effect of various factors on the kinetics of degradation," Clinical Chemistry, vol. 32, no. 2, pp. 314-319, 1986.

[41] A. Stincone, A. Prigione, T. Cramer et al., "The return of metabolism: biochemistry and physiology of the pentose phosphate pathway," Biological Reviews of the Cambridge Philosophical Society, vol. 90, no. 3, pp. 927-963, 2015.

[42] N. Pollak, C. Dölle, and M. Ziegler, "The power to reduce: pyridine nucleotides - small molecules with a multitude of functions," The Biochemical Journal, vol. 402, no. 2, pp. 205 218, 2007.

[43] J. Fan, J. Ye, J. J. Kamphorst, T. Shlomi, C. B. Thompson, and J. D. Rabinowitz, "Quantitative flux analysis reveals folatedependent NADPH production," Nature, vol. 510, no. 7504, pp. 298-302, 2014.

[44] A. Antonov, M. Agostini, M. Morello, M. Minieri, G. Melino, and I. Amelio, "Bioinformatics analysis of the serine and glycine pathway in cancer cells," Oncotarget, vol. 5, no. 22, pp. 11004-11013, 2014. 
[45] M. F. McMullin, "The molecular basis of disorders of the red cell membrane," Journal of Clinical Pathology, vol. 52, no. 4, pp. 245-248, 1999.

[46] R. B. Hamanaka and N. S. Chandel, "Warburg effect and redox balance," Science, vol. 334, no. 6060, pp. 1219-1220, 2011.

[47] S. Nóbrega-Pereira, P. J. Fernández-Marcos, T. Brioche et al., "G6PD protects from oxidative damage and improves healthspan in mice," Nature Communications, vol. 7, article 10894, 2016.

[48] K. C. Patra and N. Hay, "The pentose phosphate pathway and cancer," Trends in Biochemical Sciences, vol. 39, no. 8, pp. 347354, 2014.

[49] A. Kuehne, H. Emmert, J. Soehle et al., "Acute activation of oxidative pentose phosphate pathway as first-line response to oxidative stress in human skin cells," Molecular Cell, vol. 59, no. 3, pp. 359-371, 2015.

[50] E. L. James, R. D. Michalek, G. N. Pitiyage et al., "Senescent human fibroblasts show increased glycolysis and redox homeostasis with extracellular metabolomes that overlap with those of irreparable DNA damage, aging, and disease," Journal of Proteome Research, vol. 14, no. 4, pp. 1854-1871, 2015.

[51] E. L. James, J. A. E. Lane, R. D. Michalek, E. D. Karoly, and E. K. Parkinson, "Replicatively senescent human fibroblasts reveal a distinct intracellular metabolic profile with alterations in NAD+ and nicotinamide metabolism," Scientific Reports, vol. 6, no. 1, article 38489, 2016.

[52] R. Sullivan, "Epididymosomes: a heterogeneous population of microvesicles with multiple functions in sperm maturation and storage," Asian Journal of Andrology, vol. 17, no. 5, pp. 726-729, 2015.

[53] B. Giebel, "On the function and heterogeneity of extracellular vesicles," Annals of Translational Medicine, vol. 5, no. 6, p. 150, 2017.

[54] M. C. Larson, C. A. Hillery, and N. Hogg, "Circulating membrane-derived microvesicles in redox biology," Free Radical Biology \& Medicine, vol. 73, pp. 214-228, 2014.

[55] D. Burger, M. Turner, M. N. Munkonda, and R. M. Touyz, "Endothelial microparticle-derived reactive oxygen species: role in endothelial signaling and vascular function," Oxidative Medicine and Cellular Longevity, vol. 2016, Article ID 5047954, 10 pages, 2016.

[56] Q. Zhang, M. Shang, M. Zhang et al., "Microvesicles derived from hypoxia/reoxygenation-treated human umbilical vein endothelial cells promote apoptosis and oxidative stress in H9c2 cardiomyocytes," BMC Cell Biology, vol. 17, no. 1, p. 25, 2016.

[57] S. I. Liochev, "Reactive oxygen species and the free radical theory of aging," Free Radical Biology \& Medicine, vol. 60, pp. 1-4, 2013.

[58] J. Viña, C. Borras, K. M. Abdelaziz, R. García-Valles, and M. C. Gomez-Cabrera, "The free radical theory of aging revisited: the cell signaling disruption theory of aging," Antioxidants \& Redox Signaling, vol. 19, no. 8, pp. 779-787, 2013.

[59] A. B. Salmon, A. Richardson, and V. I. Pérez, "Update on the oxidative stress theory of aging: does oxidative stress play a role in aging or healthy aging?," Free Radical Biology \& Medicine, vol. 48, no. 5, pp. 642-655, 2010.

[60] H. Unterluggauer, B. Hampel, W. Zwerschke, and P. JansenDürr, "Senescence-associated cell death of human endothelial cells: the role of oxidative stress," Experimental Gerontology, vol. 38, no. 10, pp. 1149-1160, 2003. 


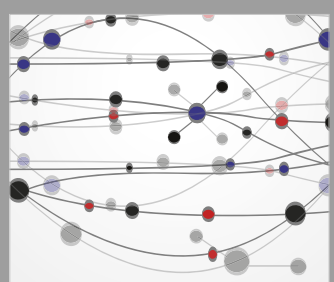

The Scientific World Journal
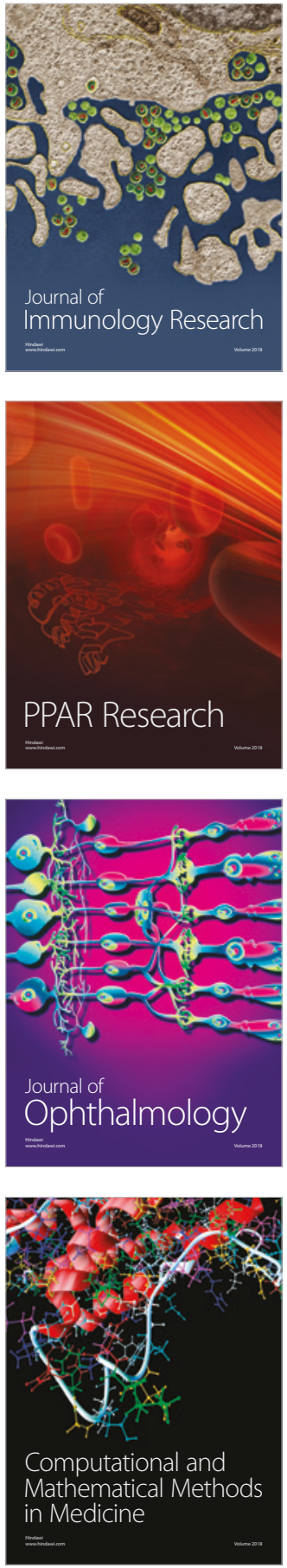

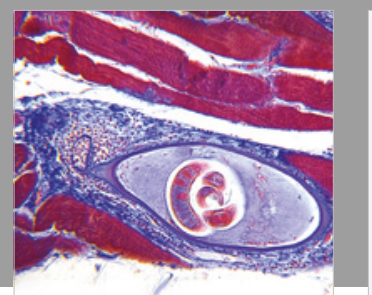

Gastroenterology Research and Practice

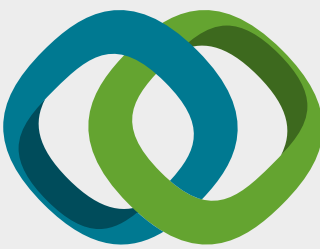

\section{Hindawi}

Submit your manuscripts at

www.hindawi.com
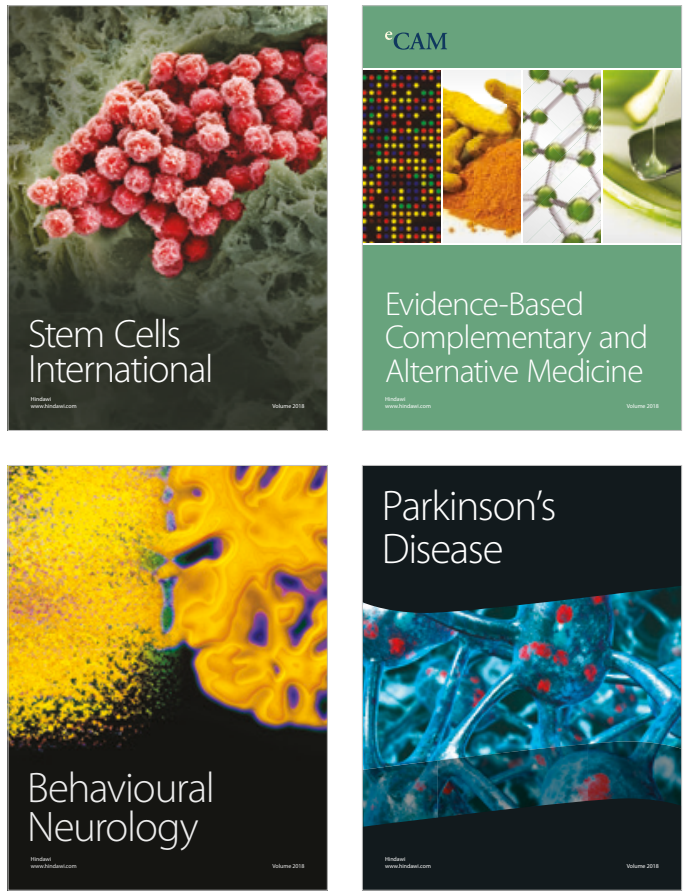

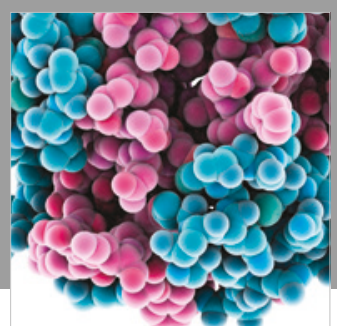

ournal of

Diabetes Research

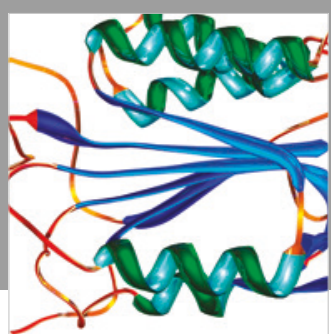

Disease Markers
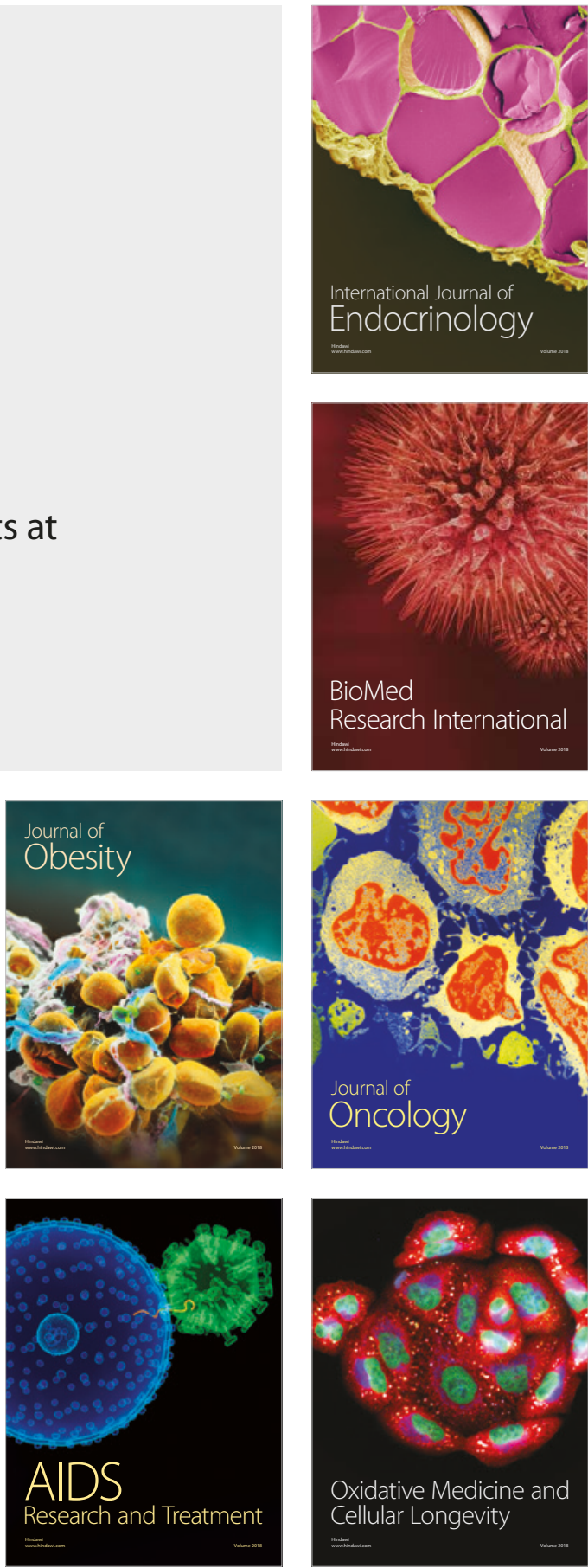